\title{
Gravitational bar detectors set limits to Planck-scale physics on macroscopic variables
}

\author{
Francesco Marin ${ }^{1,2,3 \star}$, Francesco Marino ${ }^{3,4}$, Michele Bonaldi ${ }^{5,6}$, Massimo Cerdonio ${ }^{7}$, Livia Conti ${ }^{7}$, \\ Paolo Falferi ${ }^{6,8}$, Renato Mezzena ${ }^{6,9}$, Antonello Ortolan ${ }^{10}$, Giovanni A. Prodi ${ }^{6,9}$, Luca Taffarello ${ }^{7}$, \\ Gabriele Vedovato ${ }^{7}$, Andrea Vinante ${ }^{8,11}$ and Jean-Pierre Zendri ${ }^{7}$
}

\begin{abstract}
Different approaches to quantum gravity, such as string theory ${ }^{1,2}$ and loop quantum gravity, as well as doubly special relativity ${ }^{3}$ and gedanken experiments in black-hole physics ${ }^{4-6}$, all indicate the existence of a minimal measurable length ${ }^{7,8}$ of the order of the Planck length, $L_{p}=\sqrt{\hbar G / c^{3}}=1.6 \times 10^{-35} \mathrm{~m}$. This observation has motivated the proposal of generalized uncertainty relations, which imply changes in the energy spectrum of quantum systems. As a consequence, quantum gravitational effects could be revealed by experiments able to test deviations from standard quantum mechanics ${ }^{9-11}$, such as those recently proposed on macroscopic mechanical oscillators ${ }^{12}$. Here we exploit the sub-millikelvin cooling of the normal modes of the ton-scale gravitational wave detector AURIGA, to place an upper limit for possible Planck-scale modifications on the ground-state energy of an oscillator. Our analysis calls for the development of a satisfactory treatment of multi-particle states in the framework of quantum gravity models.
\end{abstract}

General relativity and quantum physics are expected to merge at the Planck scale, defined by distances of the order of $\sim L_{\mathrm{p}}$ and/or extremely high energies of the order of $\sim E_{\mathrm{p}}=c \hbar / L_{\mathrm{p}}=1.2 \times 10^{19} \mathrm{GeV}$. Therefore, present approaches to test quantum gravitational effects are mainly focused on highenergy astronomical events ${ }^{13-15}$, which allowed stringent limits to the predicted breaking of Lorentz invariance at the Planck scale to be put in place ${ }^{16}$. On the other hand, the emergence of a minimal length scale can result in relevant consequences also for low-energy quantum mechanics experiments. The Heisenberg relation states that the uncertainties in the measurements of a position $\Delta x$ and its conjugate momentum $\Delta p$ are related by $\Delta x \Delta p \geq \hbar / 2$; that is, the position and the momentum of a particle cannot be determined simultaneously with arbitrarily high accuracy. However, an arbitrarily precise measurement of only one of the two observables, say position, is still possible at the cost of our knowledge about the other (momentum), a fact that is obviously incompatible with the existence of a minimal observable distance. This consideration motivates the introduction of generalized Heisenberg uncertainty principles ${ }^{1-7}$. As a consequence, an alternative way to check quantum gravitational effects would be to perform high-sensitivity measurements of the uncertainty relation, to reveal any possible deviation from predictions of standard quantum mechanics ${ }^{9-11}$.

In this framework, an optical experiment has recently been proposed to test quantum gravitational modifications of the canonical commutator for the variables associated with the centre of mass of a macroscopic object ${ }^{12}$. The generalization from the position/momentum of a single particle to that defining a collective motion is well described in standard quantum mechanics. The canonical commutator $[x, p]=i \hbar$ remains valid for any Lagrangian coordinate $x$ and its conjugate momentum $p$, including the coordinates describing the centre of mass or a normal vibrational mode of a macroscopic object. Recent experiments have indeed cooled down mechanical modes of micro-oscillators to their quantum ground state ${ }^{17-19}$, and in this condition the oscillators indeed exhibited peculiar quantum properties ${ }^{17,20}$. This clearly proves that a mechanical normal mode can be described by quantum-mechanical observables. On the other hand, if a modified commutator is considered, a self-consistent description in terms of macroscopic coordinates is not straightforward. Some recent works have tackled the problem, proposing solutions that imply a strong suppression of the expected effect of Planck-scale physics when probed by multi-particle objects ${ }^{21,22}$. However, none of such approaches is fully satisfactory. An experimental analysis is therefore highly desirable, in particular if performed on macroscopic variables that exhibit quantum properties.

A direct consequence of a modified commutator, when applied to the position and momentum of an harmonic oscillator, is a change in the oscillator ground-state energy $E_{\min }$ with respect to the usual $\hbar \omega_{0} / 2$. The commutators commonly proposed in quantum gravity theories ${ }^{23,24}$, giving origin to a position indetermination larger than in standard quantum mechanics (gravitational induced uncertainty), translate into a larger minimal energy. Therefore, an experiment measuring a low energy level $E_{\text {exp }}$ for a normal mode of a macroscopic system puts a straightforward upper limit to the corresponding $E_{\min }$ :

$$
E_{\min }<E_{\exp }
$$

Experimental systems particularly suitable for exploiting this relation are the cryogenic Weber bars, originally conceived and still

\footnotetext{
${ }^{1}$ Dipartimento di Fisica, Università di Firenze, Via Sansone 1, I-50019 Sesto Fiorentino (FI), Italy, ${ }^{2}$ Istituto Nazionale di Fisica Nucleare (INFN), Sezione di Firenze, Via Sansone 1, I-50019 Sesto Fiorentino (FI), Italy, ${ }^{3}$ European Laboratory for Non-Linear Spectroscopy (LENS), Via Carrara 1, I-50019 Sesto Fiorentino (FI), Italy, ${ }^{4}$ CNR-Istituto dei Sistemi Complessi, Via Madonna del Piano 10, I-50019 Sesto Fiorentino (FI), Italy, ${ }^{5}$ Institute of Materials for Electronics and Magnetism, Nanoscience-Trento-FBK Division, I-38123 Trento, Italy, ${ }^{6}$ INFN, Gruppo Collegato di Trento, I-38123 Povo, Trento, Italy, ${ }^{7}$ INFN, Sezione di Padova, via Marzolo 8, I-35131 Padova, Italy, ${ }^{8}$ Istituto di Fotonica e Nanotecnologie, CNR-Fondazione Bruno Kessler, I-38123 Povo, Trento, Italy, ${ }^{9}$ Dipartimento di Fisica, Università di Trento, I-38123 Povo, Trento, Italy, ${ }^{10}$ INFN, Laboratori Nazionali di Legnaro, I-35020 Legnaro (PD), Italy, ${ }^{11}$ Leiden Institute of Physics, Niels Bohrweg 2, 2333 CA Leiden, The Netherlands. *e-mail: marin@fi.infn.it.
} 


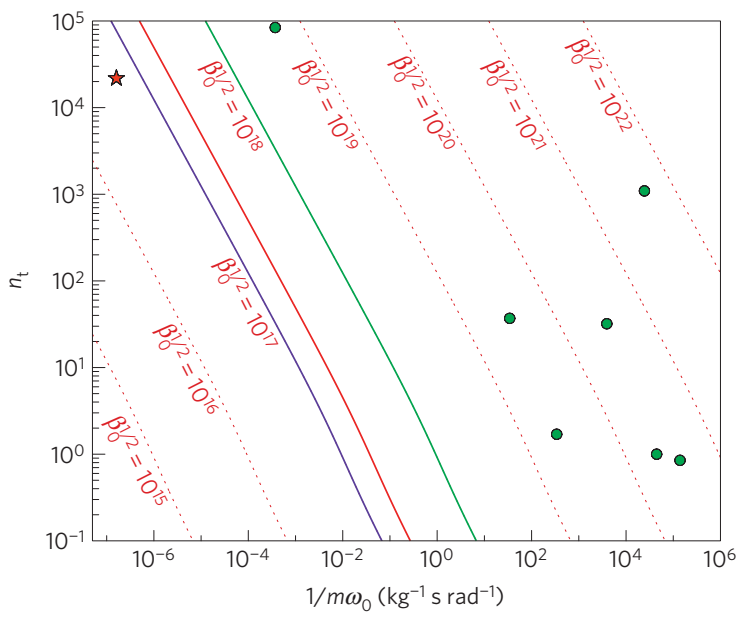

Figure 1 | Upper limits to the parameter $\beta_{0}$ that quantifies the deformation of the standard uncertainty relation in equation (2). The star corresponds to the AURIGA experiment discussed in the main text, and filled circles refer to several experiments on mechanical oscillators that are summarized in the Supplementary Information. The experimental energy $E_{\text {exp }}$ is reported on the vertical axis in terms of oscillator occupation number $n_{\mathrm{t}}$, according to $E_{\exp }=\hbar \omega_{0}\left(1 / 2+n_{\mathrm{t}}\right)$. The dashed lines are levels of $\sqrt{\beta_{0}}$, which can be interpreted as the upper limit for the length scale (normalized to the Planck length) where some new physics could come into play. Solid lines report the upper limit to $\beta_{0}$ that are given by: the lack of observed deviations from standard theory at the electroweak scale (blue line); the measurement of the Lamb shift in hydrogen (green line); the measurement of the $1 \mathrm{~S}-2 \mathrm{~S}$ level energy difference, again in hydrogen (red line)

working as detectors for gravitational waves ${ }^{25}$. They consist of large metallic bars, weighing several tons and having a main longitudinal mechanical mode oscillating around $\omega_{0} / 2 \pi \simeq 1 \mathrm{kHz}$. For our purpose, their favourable characteristics are their very large mass $\left(M \sim 10^{13}\right.$ times the Planck mass $\left.M_{\mathrm{p}}=E_{\mathrm{p}} / c^{2}=2.2 \times 10^{-8} \mathrm{~kg}\right)$; the large size of the ground-state momentum wave packet $\sqrt{\hbar M_{\text {eff }} \omega_{0}}$ associated with their main modes (where, with respect to, for example, micro-oscillators, the relatively low frequency is compensated by the large effective mass $M_{\text {eff }}$ ); and the low level of thermal energy that is reached experimentally.

Some Weber bars have been operated at ultracryogenic temperature ${ }^{26}$. However, for our purpose we focus on the AURIGA detector, whose first longitudinal mode, starting from a background bar temperature of $4.2 \mathrm{~K}$, has been further cooled using a cold damping technique down to the millikelvin regime ${ }^{27}$, a level that could be reached owing to its high mechanical quality factor. We remark that modal cooling techniques have been exploited to bring mechanical modes of micro-oscillators to their quantum ground state and that these experiments have proved that quantum behaviour of macroscopic coordinates can be obtained in this way ${ }^{17-20}$. Therefore cold damping is a valid technique for our purpose of reaching and measuring the lowest oscillator modal energy, even if such modal cooling cannot be exploited to increase the sensitivity of the oscillating system as a detector of external excitation.

In AURIGA, the modal motion is measured by coupling the bar to two further oscillators: the first is a mechanical one, having the same frequency but a lower mass, and working as a resonant mechanical amplifier read by a capacitive transducer; the second one is a nearly resonant electrical LC circuit coupled to a d.c. superconducting quantum interference device amplifier ${ }^{28,29}$. All of the three oscillators have been cooled down by cold damping, to roughly the same temperature. A detailed analysis of the system shows that a minimal energy of $1.3 \times 10^{-26} \mathrm{~J}$ can be attributed to the first longitudinal mode of the bar, whose resonance frequency is $\omega_{0} / 2 \pi=900 \mathrm{~Hz}$ (ref. 27). The motion of this mode is symmetrical with respect to the plane, perpendicular to the bar axis, that bisects the bar, and implies an oscillation of the centre-of-mass of each half-bar. The reduced mass of this couple of centre-of-masses is $M_{\text {red }}=M / 2=1.1 \times 10^{3} \mathrm{~kg}$ (where $M$ is the bar physical mass), and the energy associated with the oscillation of the centre-of-masses is $\sim 80 \%$ of the total modal energy. The measured modal energy $E_{\exp }$ is therefore an upper limit also for the minimal energy $E_{\min }$ of the oscillation of the centre-of-masses and it can be used in equation (1) and in the relations that follow from it (a detailed description of the AURIGA detector, of the minimal measured energy and a discussion on the appropriate value of the mass to be used in this analysis are reported in the Supplementary Information).

We start our analysis from the uncertainty relation ${ }^{1,2,7}$

$$
\Delta x \Delta p \geq \frac{\hbar}{2}\left(1+\beta_{0}\left(\frac{\Delta p}{M_{\mathrm{p}} c}\right)^{2}\right)
$$

where $\beta_{0}$ is a dimensionless parameter that should be around unity if the modification is efficient at the Planck scale. If $\beta_{0}$ is larger than unity, it defines a new length scale $\sqrt{\beta_{0}} L_{\mathrm{p}}$ where some new physics should come into play ${ }^{9}$. Normalizing the coordinate and momentum to their ground-state wave-packet size according to $x=\sqrt{\left(\hbar / m \omega_{0}\right)} X$ and $p=\sqrt{\hbar m \omega_{0}} P$, the Hamiltonian operator for a harmonic oscillator is written as

$$
H=\frac{\hbar \omega_{0}}{2}\left(X^{2}+P^{2}\right)
$$

and the uncertainty relation given in equation (2) becomes

$$
\Delta X \Delta P \geq \frac{1}{2}\left(1+\beta(\Delta P)^{2}\right)
$$

where $\beta=\beta_{0}\left(\hbar m \omega_{0} / M_{\mathrm{p}}^{2} c^{2}\right)$. The minimal oscillator energy is found for $\langle X\rangle=\langle P\rangle=0$ using the equality in equation (4). Extracting $\Delta X$ from equation (4) and inserting it in equation (3) we obtain

$$
E>\frac{\hbar \omega_{0}}{2}\left[\left(1+\frac{\beta^{2}}{4}\right)(\Delta P)^{2}+\frac{1}{4(\Delta P)^{2}}+\frac{\beta}{2}\right]
$$

and minimizing with respect to $\Delta P$ we find the minimal energy

$$
E_{\min }=\frac{\hbar \omega_{0}}{2}\left[\sqrt{1+\frac{\beta^{2}}{4}}+\frac{\beta}{2}\right] \simeq \frac{\hbar \omega_{0}}{2} \beta
$$

where the last approximation (which simplifies the form but is not indeed necessary) is valid for $\beta \gg 1$. Comparing this expression of $E_{\min }$ with the measured $E_{\exp }$, according to equation (1), we obtain $\beta<\left(2 E_{\exp } / \hbar \omega_{0}\right)$, which for $\beta_{0}$ can be written in the meaningful form

$$
\beta_{0}<2 \frac{E_{\exp }}{\hbar \omega_{0}} \frac{M_{\mathrm{p}}}{m} \frac{M_{\mathrm{p}} c^{2}}{\hbar \omega_{0}}
$$

We now come back to the first longitudinal mode of AURIGA with cold damping, and consider the oscillation of the couple of centre-of-masses of the half-bars. Using the mode resonance frequency, the reduced mass $M_{\text {red }}$ and the measured modal energy $E_{\text {exp }}=1.3 \times 10^{-26} \mathrm{~J}$, we obtain $\beta<4.4 \times 10^{4}$ and $\beta_{0}<3 \times 10^{33}$. Our upper limit for $\beta_{0}$ is still far from forbidding new physics at the Planck scale. It can be compared to similar limits of $\beta_{0}<10^{34}$, imposed by the lack of observed deviations from standard theory at the electroweak scale; $\beta_{0}<10^{36}$, calculated from the accurate measurement of the Lamb shift in hydrogen ${ }^{9}$ and $\beta_{0}<4 \times 10^{34}$ 
from the 1S-2S level energy difference, again in hydrogen ${ }^{22}$. These limits are summarized in Fig. 1, together with those obtainable with the same method from other experiments with cold macroscopic oscillators (listed in the Supplementary Information).

In analysing the consequence of generalized Heisenberg uncertainty principles on the foreseen behaviour of a macroscopic object, the preliminary assumption that the modified rules can be applied to macroscopic coordinates must be carefully taken into account. With this consideration in mind, our experimental analysis has a double interpretation. On one side, it sets interesting limits to the Planck-scale physics, and helps to compare the consequences of various approaches to quantum gravity. On the other side, it strongly calls for an effort in developing theories that offer a reasonable path from basic properties of spacetime geometry and of the measurement process (that is, main issues of the theories that should merge into quantum gravity) to multi-particle and macroscopic reality. This research field, which suffers from poor experimental feedback, is likely to benefit from precise metrological systems, including for example interferometric gravitational wave detectors $^{25,30,31}$, whose data are already the subject of analyses focused on quantum gravity effects ${ }^{32,33}$, and ultracryogenic devices that could in the near future include, owing to dedicated experiments, oscillators with mass of the order of, or larger than, $M_{\mathrm{p}}$ operating in their fundamental quantum state ${ }^{27}$.

Received 4 June 2012; accepted 9 November 2012; published online 16 December 2012

\section{References}

1. Amati, D., Ciafaloni, M. \& Veneziano, G. Superstring collisions at Planckian energies. Phys. Lett. B 197, 81-88 (1987).

2. Gross, D. J. \& Mende, P. F. String theory beyond the Planck scale. Nucl. Phys. B 303, 407-454 (1988).

3. Amelino-Camelia, G. Doubly special relativity: First results and key open problems. Int. J. Mod. Phys. D 11, 1643-1669 (2002).

4. Maggiore, M. A generalized uncertainty principle in quantum gravity. Phys. Lett. B 304, 65-69 (1993).

5. Scardigli, F. Generalized uncertainty principle in quantum gravity from micro-black hole gedanken experiment. Phys. Lett. B 452, 39-44 (1999).

6. Jizba, P., Kleinert, H. \& Scardigli, F. Uncertainty relation on a world crystal and its applications to micro black holes. Phys. Rev. D 81, 084030 (2010).

7. Garay, L. G. Quantum gravity and minimum length. Int. J. Mod. Phys. A 10, 145-165 (1995).

8. Hossenfelder, S. Minimal length scale scenarios for quantum gravity. Living Rev. Relativ. Preprint at http://arxiv.org/abs/arXiv:1203.6191 (2012).

9. Das, S. \& Vagenas, E. C. Universality of quantum gravity corrections. Phys. Rev. Lett. 101, 221301 (2008).

10. Ali, A. F., Das, S. \& Vagenas, E. C. Discreteness of space from the generalized uncertainty principle. Phys. Lett. B 678, 497-499 (2009).

11. Ali, A. F., Das, S. \& Vagenas, E. C. A proposal for testing quantum gravity in the lab. Phys. Rev. D 84, 044013 (2011).

12. Pikovski, I., Vanner, M. R., Aspelmeyer, M., Kim, M. S. \& Brukner, Č. Probing Planck-scale physics with quantum optics. Nature Phys. 8, 393-397 (2012).

13. Amelino-Camelia, G., Ellis, J., Mavromatos, N. E., Nanopoulos, D. V. \& Sarkar, S. Tests of quantum gravity from observations of gamma-ray bursts. Nature 393, 763-765 (1998).
14. Jacob, U. \& Piran, T. Neutrinos from gamma-ray bursts as a tool to explore quantum-gravity-induced Lorentz violation. Nature Phys. 7, 87-90 (2007).

15. Tamburini, F., Cuofano, C., Della Valle, M. \& Gilmozzi, R. No quantum gravity signature from the farthest quasars. Astron. Astrophys. 533, 1-5 (2011).

16. Abdo, A. A. et al. A limit on the variation of the speed of light arising from quantum gravity effects. Nature 462, 331-334 (2009).

17. O'Connell, A. D. et al. Quantum ground state and single-phonon control of a mechanical resonator. Nature 464, 697-713 (2010).

18. Teufel, J. D. et al. Sideband cooling of micromechanical motion to the quantum ground state. Nature 475, 359-363 (2011)

19. Chan, J. et al. Laser cooling of a nanomechanical oscillator into its quantum ground state. Nature 478, 89-92 (2011).

20. Safavi-Naeini, A. H. et al. Observation of quantum motion of a nanomechanical resonator. Phys. Rev. Lett. 108, 033602 (2012).

21. Hossenfelder, S. Multiparticle states in deformed special relativity. Phys. Rev. D 75, 105005 (2007).

22. Quesne, C. \& Tkachuk, V. M. Composite system in deformed space with minimal length. Phys. Rev. A 81, 012106 (2010).

23. Maggiore, $M$. The algebraic structure of the generalized uncertainty principle. Phys. Lett. B 319, 83-86 (1993).

24. Kempf, A., Mangano, G. \& Mann, R. B. Hilbert space representation of the minimal length uncertainty relation. Phys. Rev. D 52, 1108-1118 (1995).

25. Sathyaprakash, B.S. \& Schutz, B. F. Physics, astrophysics and cosmology with gravitational waves. Living Rev. Relativ. 12, 1-141 (2009).

26. Allen, Z. A. et al. First search for gravitational wave bursts with a network of detectors. Phys. Rev. Lett. 85, 5046-5050 (2000).

27. Vinante, A. et al. Feedback cooling of the normal modes of a massive electromechanical system to submillikelvin temperature. Phys. Rev. Lett. 101, 033601 (2008).

28. Vinante, A. et al. Stabilization and optimization of a two-stage d.c. SQUID coupled to a high Q resonator. Physica C 368, 176-180 (2002).

29. Baggio, L. et al. 3-mode detection for widening the bandwidth of resonant gravitational wave detectors. Phys. Rev. Lett. 94, 241101 (2005).

30. Selected Articles from the 9th Edoardo Amaldi Meeting and the 2011 Numerical Relativity and Data Analysis Meeting (AMALDI 9/NRDA 2011) Class. Quant. Grav. 29, 120301-129603 (Special issue, 2012).

31. Cerdonio, M. \& Losurdo, G. Gravitational waves: from discovery to astronomy. La Riv. Nuovo Cimento 35, 389-480 (2012).

32. Amelino-Camelia, G. Gravity-wave interferometers as quantum-gravity detectors. Nature 398, 216-218 (1999).

33. Hogan, C. J. Interferometers as probes of Planckian quantum geometry. Phys. Rev. D 85, 064007 (2012).

\section{Acknowledgements}

F. Marin and F. Marino thank D. Seminara and M. Inguscio for useful discussions.

\section{Author contributions}

F. Marin conceived the work, which was further developed with F. Marino and all other authors. F. Marin and F. Marino wrote the manuscript with input from all of the authors.

\section{Additional information}

Supplementary information is available in the online version of the paper. Reprints and permissions information is available online at www.nature.com/reprints.

Correspondence and requests for materials should be addressed to F. Marin

\section{Competing financial interests}

The authors declare no competing financial interests. 